\title{
Flaw Detection in Layered Media Based on Parametric Modeling of Overlapping Ultrasonic Echoes
}

\author{
Fredrik Hägglund* (student member), Jesper Martinsson, (student member), and Johan E. Carlson, (member) \\ EISLAB, Dept. of Computer Science and Electrical Engineering, Luleå University of Technology, SE-971 87 Luleå, Sweden. \\ *Email: Fredrik.Hagglund@ltu.se
}

\begin{abstract}
In materials consisting of several thin layers, multiple reflections within the structure give rise to received ultrasonic signals composed of overlapping echoes. In this paper we present a parametric model that can be used to decompose such signals into the individual reflections. We derive a Maximum Likelihood Estimator for the the model parameters, which are then used in a Generalized Likelihood Ratio Test (GLRT) to detect flaws in multi-layered structures. We show with simulations how the presence of a thin bonding layer in a three-layer structure can be detected. The probability of detection is shown to be $\approx 96 \%$, for a signal-to-noise ratio (SNR) of $15 \mathrm{~dB}$ and a probability of false alarm of $5 \%$.
\end{abstract}

\section{INTRODUCTION}

Non-destructive testing of materials using ultrasound has a wide range of applications in the area of process control, see for example [1], [2] and [3]. In this paper we propose a detector, based on parametric modeling of the received ultrasound waveform, to be used for flaw detection in layered media. The model structure is chosen so that all dynamics of the waveform is captured by a small number of parameters. All subsequent analysis is then done on the model parameters rather than on the entire waveform, which enables costeffective storage and fast processing.

The material is modeled using a continuous autoregressive (AR) model [4], [5] with parameters connected to physical properties, related to the thicknesses of the material layers and the reflection coefficients given by the layer boundaries. In this paper we derive a general model structure for reflections from layered media. We then show with simulations how the Maximum Likelihood Estimate (MLE) of the parameters can be used to reconstruct the waveform.

The task of the detector is to determine whether a thin bonding layer in a three-layer structure is present or not. The detection scheme is based on the Generalized Likelihood Ratio Test (GLRT) which, given the MLEs of the model parameters, yields the detection rule.

The detection scheme is verified using simulations on threelayered materials, where two thin $0.5-5 \mathrm{~mm}$ layers are bonded together by a $20-50 \mu \mathrm{m}$ layer. Results show the evaluation of the probability of detection vs the probability of false alarm for varying signal-to-noise ratios and layer thicknesses. The evaluation of the detector threshold gives the probability of detection for varying situations.

\section{Signal Model}

Sending an ultrasonic echo through a multi-layered material will produce a received output signal waveform consisting of several overlapping, delayed and attenuated echoes. The multi-layered structure is modeled using a continuous AR model, where the AR coefficients are connected directly to the physical properties of the material. For a single layer the AR model is relatively simple, but expands as the number of layers increases. The effects of dispersion and diffraction are assumed to be negligible and the different materials are considered to be lossless. Hence, the material attenuation is not included in the model in this paper, but could be easily modeled using an additional parameter for each layer.

\section{A. Model for a Single Layer}

Considering the ultrasonic wave to be a harmonic plane wave in the far field [6], equations for waves in layered media can be used. If the incident wave is perpendicular to the surface, the density and sound velocity forms the reflection and transmission coefficients used to calculate the amplitude of the reflected waves. The time of arrival of an ultrasonic echo traveling back and forward in a layer is represented by $\tau=\frac{2 d_{q}}{c_{q}}$, where $c_{q}$ is the speed of sound and $d_{q}$ is the thickness of layer $q$, respectively.

Assume a model structure shown in Eq. (1), where $\mathrm{U}(\omega)$ is the input signal in the frequency domain and $\mathrm{Y}(\omega)$ is the received output signal in the frequency domain. The model of the transfer function is a reverberant multi-layered structure $\mathrm{H}(\omega)$. The output $\mathrm{Y}(\omega)$ is then represented as

$$
\mathrm{Y}(\omega)=\mathrm{H}(\omega) \mathrm{U}(\omega)
$$

where

$$
\mathrm{H}(\omega)=\left(R_{01}+\mathrm{G}(\omega)\right) e^{-j \omega \tau_{0}}
$$

where $\omega=2 \pi f$ ( $f$ being the frequency of the sound wave) and $R_{01}$ is the reflection coefficient from the top layer and $\tau_{0}$ is the time delay for the ultrasonic pulse traveling the distance $d_{0}$ back and forward from the transducer to the material in the water buffer region. The function $G(\omega)$ in Eq. (2) is the general model of the multi-layered structure for $q$ layers. An iterative model for the reflection from a multi-layered structure has been derived by Brekhovskikh [7], but is here derived in a more convenient way for system transfer function 
identification. In Fig. 1 the model is shown for a one-layer structure. In [7] it is shown that for a one-layer structure, ignoring $R_{01}$, the reflection from within the layer is

$$
\begin{aligned}
R & =T_{01} R_{12} T_{10} e^{-j \omega \tau_{1}} \sum_{n=0}^{\infty}\left(R_{10} R_{12} e^{-j \omega \tau_{1}}\right)^{n} \\
& =\frac{T_{01} R_{12} T_{10} e^{-j \omega \tau_{1}}}{1-R_{10} R_{12} e^{-j \omega \tau_{1}}}
\end{aligned}
$$

The first coefficient, $R_{01}$, can be ignored because it is already in our model in Eq. (2). The inequality $\left|R_{10} R_{12} e^{-j \omega \tau_{1}}\right|<1$ must be fulfilled for the system to be stable. This condition is assumed to hold for each sum of echoes following in the derivation of the signal model. Using Eq. (3) in the transfer function $H(\omega)$ in Eq. (2) as $G(\omega)$ gives the transfer function for a one-layer structure. This holds for an arbitrary number of layers and hence, $\mathrm{G}_{q}(\omega)$ is the model for the multi-layered structure of $q$ layers in the transfer function $\mathrm{H}_{q}(\omega)$.

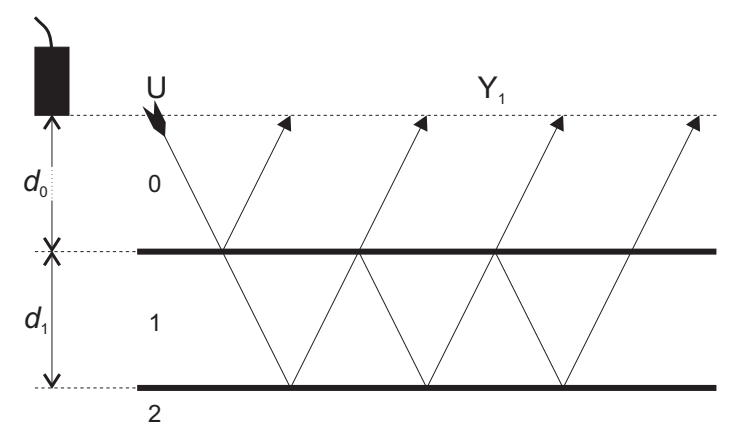

Fig. 1. A one-layer structure with the input $U(\omega)$ and the output signal waveform $Y_{1}(\omega)$.

\section{B. Model for an Arbitrary Number of Layers}

Every single layer in the multi-layered structure can be viewed as the layer in Fig. 2 with the summed inputs from both overlying and underlying layers.

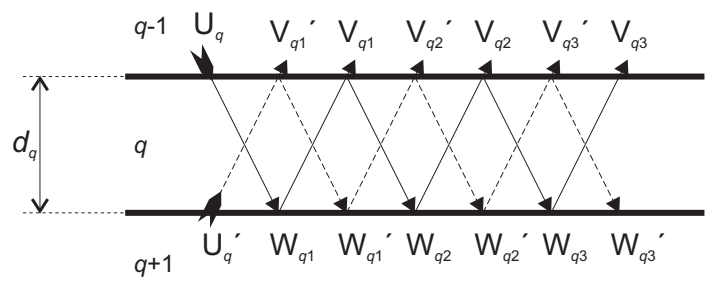

Fig. 2. The $q$ :th layer with the inputs $\mathrm{U}_{q}(\omega)$ and $\mathrm{U}_{q}(\omega)^{\prime}$, and the echoes that contributes to the outputs at the boundaries of the layer.

The output signal at the boundary of the top of layer $q$ is the sum of all the echoes $\mathrm{V}_{q i}$ and $\mathrm{V}_{q i}^{\prime}$, i.e.

$$
\mathrm{U}_{q-1}^{\prime}=A_{q} \mathrm{U}_{q}+B_{q} \mathrm{U}_{q}^{\prime},
$$

where

$$
\begin{aligned}
A_{q} & =\frac{T_{q-1, q} R_{q, q+1} T_{q, q-1} e^{-j \omega \tau_{q}}}{1-R_{q, q-1} R_{q, q+1} e^{-j \omega \tau_{q}}} \\
B_{q} & =\frac{T_{q, q-1} e^{-j \omega \frac{1}{2} \tau_{q}}}{1-R_{q, q-1} R_{q, q+1} e^{-j \omega \tau_{q}}} .
\end{aligned}
$$

This signal, $\mathrm{U}_{q-1}^{\prime}$, is the input signal at the boundary to the overlying layers. The output signal at the boundary of the bottom of the layer $q$ is the sum of all the echoes $\mathrm{W}_{q i}$ and $\mathrm{W}_{q i}^{\prime}$, i.e.

$$
\mathrm{U}_{q+1}=C_{q} \mathrm{U}_{q}+D_{q} \mathrm{U}_{q}^{\prime}
$$

where

$$
\begin{aligned}
C_{q} & =\frac{T_{q-1, q} e^{-j \omega \frac{1}{2} \tau_{q}}}{1-R_{q, q-1} R_{q, q+1} e^{-j \omega \tau_{q}}} \\
D_{q} & =\frac{R_{q, q-1} e^{-j \omega \tau_{q}}}{1-R_{q, q-1} R_{q, q+1} e^{-j \omega \tau_{q}}} .
\end{aligned}
$$

The output of this layer, $\mathrm{U}_{q+1}$, is the input at the boundary to the underlying layers. If we have $q$ layers in a structure, $\mathrm{U}_{q+1}$ is not needed since it is the output of the entire structure and no further reflections will be possible.

\section{The General Multi-Layered Structure}

The model for an entire multi-layered structure can be derived as

$$
\mathrm{G}_{q}(\omega)=A_{q-1}+\frac{B_{q-1} C_{q-1} \mathrm{G}_{q+1}(\omega)}{1-D_{q-1} \mathrm{G}_{q+1}(\omega)},
$$

for $q \geq 2$. The case when $q=1$ was derived in Eq. (3) and with these notations it is $\mathrm{G}_{1}(\omega)=A_{1}$. For $q$ layers the model is extended using the formula in Eq. (10), and finally, $\mathrm{G}_{q+1}(\omega)$, is equal to $A_{q}$ for $q$ layers, where $A_{q}$ is the amplitude of the $q$ :th reflection.

\section{Transfer Function Models for Two and Three Layers}

Here we state the explicit expressions for the transfer functions for the cases of two and three layers. The general model of the multi-layered structure $\mathrm{G}_{q}(\omega)$ is derived for two and three layers and inserted in the transfer function $\mathrm{H}_{q}(\omega)$, in Eq. (2). For a two-layer structure the entire model is

$$
\begin{aligned}
\mathrm{H}_{2}(\omega)= & R_{01} e^{-j \omega \tau_{0}}+\left(1-R_{01}^{2}\right) e^{-j \omega\left(\tau_{0}+\tau_{1}\right)} \\
& \left(R_{12}+R_{23} e^{-j \omega \tau_{2}}\right) \cdot \\
& \left(1+R_{01} R_{12} e^{-j \omega \tau_{1}}+R_{12} R_{23} e^{-j \omega \tau_{2}}\right. \\
& \left.+R_{01} R_{23} e^{-j \omega\left(\tau_{1}+\tau_{2}\right)}\right)^{-1}
\end{aligned}
$$

with $\boldsymbol{\theta}_{1}=\left[\begin{array}{llllll}R_{01} & R_{12} & R_{23} & \tau_{0} & \tau_{1} & \tau_{2}\end{array}\right]^{T}$ as the vector of parameters to be estimated. For a three-layer structure the corresponding model is

$$
\begin{aligned}
\mathrm{H}_{3}(\omega)= & R_{01} e^{-j \omega \tau_{0}}+\left(1-R_{01}^{2}\right) e^{-j \omega\left(\tau_{0}+\tau_{1}\right)} \\
& \left(R_{12}+R_{23} e^{-j \omega \tau_{2}}+R_{12} R_{23} R_{34} e^{-j \omega \tau_{3}}\right. \\
& \left.+R_{34} e^{-j \omega\left(\tau_{2}+\tau_{3}\right)}\right) \cdot \\
& \left(1+R_{01} R_{12} e^{-j \omega \tau_{1}}+R_{12} R_{23} e^{-j \omega \tau_{2}}\right. \\
& +R_{23} R_{34} e^{-j \omega \tau_{3}}+R_{01} R_{12} R_{23} R_{34} e^{-j \omega\left(\tau_{1}+\tau_{3}\right)} \\
& +R_{12} R_{34} e^{-j \omega\left(\tau_{2}+\tau_{3}\right)}+R_{01} R_{23} e^{-j \omega\left(\tau_{1}+\tau_{2}\right)} \\
& \left.+R_{01} R_{34} e^{-j \omega\left(\tau_{1}+\tau_{2}+\tau_{3}\right)}\right)^{-1}
\end{aligned}
$$

with $\boldsymbol{\theta}_{0}=\left[\begin{array}{llllllll}R_{01} & R_{12} & R_{23} & R_{34} & \tau_{0} & \tau_{1} & \tau_{2} & \tau_{3}\end{array}\right]^{T}$. 


\section{Parameter Estimation}

Given the model structure described in Section II, we here derive the frequency domain Maximum Likelihood Estimator (MLE) for the model parameters. From Eq. (1) we have

$$
\mathbf{Y}(\boldsymbol{\omega})=\mathbf{H}(\boldsymbol{\omega}, \boldsymbol{\theta}) \mathbf{U}(\boldsymbol{\omega})
$$

where both the input signals $\mathbf{U}(\boldsymbol{\omega})$ and the output signals $\mathbf{Y}(\boldsymbol{\omega})$ are corrupted by measurement noise and $\boldsymbol{\theta}$ is the parameter vector. The input signals $\mathbf{U}(\boldsymbol{\omega})$ and the output signals $\mathbf{Y}(\boldsymbol{\omega})$ are $M \times N$ matrices of $M$ measurements with $N$ samples each. The measurement noise is assumed to be white and Gaussian. The scaled error of the model is

$$
\varepsilon(\boldsymbol{\omega}, \boldsymbol{\theta})=\frac{\mathbf{Y}(\boldsymbol{\omega})-\mathbf{H}(\boldsymbol{\omega}, \boldsymbol{\theta}) \mathbf{U}(\boldsymbol{\omega})}{\boldsymbol{\sigma}_{e}(\boldsymbol{\omega}, \boldsymbol{\theta})}
$$

where $\boldsymbol{\sigma}_{e}^{2}(\boldsymbol{\omega}, \boldsymbol{\theta})$ is the variance of the error [8], given by

$$
\boldsymbol{\sigma}_{e}^{2}(\boldsymbol{\omega}, \boldsymbol{\theta})=\boldsymbol{\sigma}_{y}^{2}+\boldsymbol{\sigma}_{u}^{2}|\mathbf{H}(\boldsymbol{\omega}, \boldsymbol{\theta})|^{2}-2 \Re\left\{\boldsymbol{\sigma}_{y u}^{2} \mathbf{H}(\boldsymbol{\omega}, \boldsymbol{\theta})\right\}
$$

If $\boldsymbol{\theta}$ is the unknown parameter vector and $\mathbf{C}_{e}$ is the covariance matrix of the error, the multivariate complex Gaussian PDF becomes

$$
p(\varepsilon ; \boldsymbol{\theta})=\left(\pi^{N} \operatorname{det}\left(\mathbf{C}_{e}\right)\right)^{-1} e^{-\boldsymbol{\varepsilon}(\boldsymbol{\theta})^{H} \boldsymbol{\varepsilon}(\boldsymbol{\theta})} .
$$

The derivative of the log-likelihood function being set to zero minimizes the error. The Gauss-Newton method is then used to iteratively find the parameter vector [9], i.e.

$$
\boldsymbol{\theta}_{k+1}=\boldsymbol{\theta}_{k}-\left(\Re\left\{\frac{\partial \boldsymbol{\varepsilon}^{H}}{\partial \boldsymbol{\theta}_{k}} \frac{\partial \boldsymbol{\varepsilon}}{\partial \boldsymbol{\theta}_{k}}\right\}\right)^{-1} \cdot \Re\left\{\frac{\partial \boldsymbol{\varepsilon}^{H}}{\partial \boldsymbol{\theta}_{k}} \varepsilon\right\} .
$$

\section{Flaw Detector}

A detector may be thought of as a mapping from the data values into a decision, or in our case from the parameters into a decision. A Generalized Likelihood Ratio Test (GLRT) is a hypothesis test used in the detection algorithm, with the advantage that the detection is performed on the MLEs of the parameters. The task for the detector is to determine whether the modeled material has a two- or a three-layered structure, using the estimated parameter vector $\hat{\boldsymbol{\theta}}$. The hypothesis in the test can be stated as

$$
\begin{aligned}
& \mathcal{H}_{0}: \mathrm{H}(\hat{\boldsymbol{\theta}})=\mathrm{H}_{3}\left(\hat{\boldsymbol{\theta}}_{0}\right), \\
& \mathcal{H}_{1}: \mathrm{H}(\hat{\boldsymbol{\theta}})=\mathrm{H}_{2}\left(\hat{\boldsymbol{\theta}}_{1}\right),
\end{aligned}
$$

where the null hypothesis, $\mathcal{H}_{0}$, is the case when there are a three-layered structure. The GLRT is not associated with any optimality criterion, but is nevertheless commonly used. The GLRT decides hypothesis $\mathcal{H}_{1}$ if [10]

$$
L_{G}(\varepsilon)=\frac{p\left(\varepsilon ; \hat{\boldsymbol{\theta}}_{1}, \mathcal{H}_{1}\right)}{p\left(\varepsilon ; \hat{\boldsymbol{\theta}}_{0}, \mathcal{H}_{0}\right)}>\gamma
$$

where $\hat{\boldsymbol{\theta}}_{1}$ is the MLE of $\boldsymbol{\theta}_{1}$ and $\hat{\boldsymbol{\theta}}_{0}$ is the MLE of $\boldsymbol{\theta}_{0}$. The MLEs of $\boldsymbol{\theta}_{1}$ and $\boldsymbol{\theta}_{0}$ are found by maximizing Eq. (16) with respect to $\boldsymbol{\theta}_{1}$ and $\boldsymbol{\theta}_{0}$, respectively, where the estimated covariance matrix of the error $\hat{\mathbf{C}}_{e}$ is used. Using the MLEs of $\boldsymbol{\theta}_{1}$ and $\boldsymbol{\theta}_{0}$ in Eq. (16) the likelihood ratio in Eq. (19) is

$$
L_{G}(\boldsymbol{\varepsilon})=\frac{\frac{1}{\pi^{N} \operatorname{det}\left(\hat{\mathbf{C}}_{e}\right)} e^{-\boldsymbol{\varepsilon}\left(\hat{\boldsymbol{\theta}}_{1}\right)^{H} \boldsymbol{\varepsilon}\left(\hat{\boldsymbol{\theta}}_{1}\right)}}{\frac{1}{\pi^{N} \operatorname{det}\left(\hat{\mathbf{C}}_{e}\right)} e^{-\boldsymbol{\varepsilon}\left(\hat{\boldsymbol{\theta}}_{0}\right)^{H} \boldsymbol{\varepsilon}\left(\hat{\boldsymbol{\theta}}_{0}\right)}} .
$$

Simplifying and taking the logarithms on both sides we have

$$
\ln L_{G}(\boldsymbol{\varepsilon})=\mathbf{V}\left(\hat{\boldsymbol{\theta}}_{0}\right)-\mathbf{V}\left(\hat{\boldsymbol{\theta}}_{1}\right)>\ln \gamma=\gamma^{\prime}
$$

where $\mathbf{V}(\hat{\boldsymbol{\theta}})=\boldsymbol{\varepsilon}(\hat{\boldsymbol{\theta}})^{H} \boldsymbol{\varepsilon}(\hat{\boldsymbol{\theta}})$ is the cost function. Hence, we decide $\mathcal{H}_{1}$ if

$$
\mathbf{V}\left(\hat{\boldsymbol{\theta}}_{0}\right)-\mathbf{V}\left(\hat{\boldsymbol{\theta}}_{1}\right)>\gamma^{\prime} .
$$

The threshold $\gamma$ is decided from

$$
P_{F A}=\int_{\{\boldsymbol{\varepsilon} ; L(\boldsymbol{\varepsilon})>\gamma\}} p\left(\varepsilon ; \hat{\boldsymbol{\theta}}_{0}, \mathcal{H}_{0}\right)=\alpha
$$

where $P_{F A}$ is the probability of false alarm and $\alpha$ is the level of significance of the test.

\section{Simulations}

The signal model and the detector are evaluated through a simulation procedure to be able to show the precision in the parameter estimation and the efficiency in the detection algorithm. The performance is evaluated in a simulation environment consisting of a three-layer structure where the middle layer is a thin bonding layer. The task for the detector is to determine when the bonding layer is missing. To simulate the signal model a Gaussian echo [11] $s(t)=$ $\beta e^{-\alpha(t-\tau)^{2}} \cos \left(2 \pi f_{c}(t-\tau)+\phi\right)$, with $\beta=1, \alpha=1500(\mathrm{MHz})^{2}$, $\tau=1 \mu \mathrm{s}, f_{c}=35 \mathrm{MHz}$ and $\phi=1 \mathrm{rad}$, is used as the input time domain reference signal. The simulated data consists of 2048 points with a sampling rate $f_{s}$ of $400 \mathrm{MHz}$. AR models with two and three layers were simulated to create output signals, and measurement noise in the frequency domain with varying signal-to-noise ratio (SNR) were added to the input and output signal. If $P_{\text {noise }}=\sigma^{2}$ is the variance of the noise in the frequency domain, and $P_{\text {signal }}=\sigma_{U}^{2}$ is the variance of the input signal $U$, the SNR is defined as

$$
\mathrm{SNR}=10 \log \frac{P_{\text {signal }}}{P_{\text {noise }}}
$$

The parameters used when creating the AR models were for two layers, $\boldsymbol{\theta}=\left[\begin{array}{llllll}0.7 & -0.3 & -0.5 & 0 & 286 & 38\end{array}\right]^{T}$ and for three layers $\boldsymbol{\theta}=\left[\begin{array}{llllllll}0.7 & -0.4 & 0.1 & -0.5 & 0 & 286 & 5 & 33\end{array}\right]^{T}$. These parameters are directly connected to physics. The first half in the parameter vectors are the reflection coefficients between the layers and the second half are the sample times of arrival, $\tau_{s}=f_{s} \tau$, associated to the thickness of the layers. In the threelayer case the top layer has the highest acoustic impedance and the middle bonding layer has the lowest acoustic impedance. In the two-layer case the middle bonding layer is missing. The thickness of the top layer is $2 \mathrm{~mm}$, the middle layer $30 \mu \mathrm{m}$ and the bottom layer $0.5 \mathrm{~mm}$. These choices of the layer thicknesses produces overlapping echoes in the simulated output signal.

The estimation is performed with the starting values directly connected to the theoretical values of the reflection coefficients and the layer thickness. In the three-layer case the starting values of the parameter vector is $\boldsymbol{\theta}=\left[\begin{array}{llll}0.73 & -0.39 & 0.08 & -\end{array}\right.$ $\left.\begin{array}{lllll}0.53 & 0 & 286 & 5 & 33\end{array}\right]^{T}$ and in the two-layer case, $\boldsymbol{\theta}=\left[\begin{array}{lll}0.73 & -\end{array}\right.$ $\left.\begin{array}{lllll}0.32 & -0.53 & 0 & 286 & 38\end{array}\right]^{T}$. 


\section{RESULTS}

The evaluation of the parameter estimation shows that a reverberant overlapping signal waveform can be estimated using the parametric model described in this paper. In Fig. 3 the estimated signal waveform is plotted along with the simulated waveform. The simulation environment produces overlapping echoes to test the parameter estimation. Since the residuals are consisting of noise only, the signal waveform can be described by the estimated parameter vector.
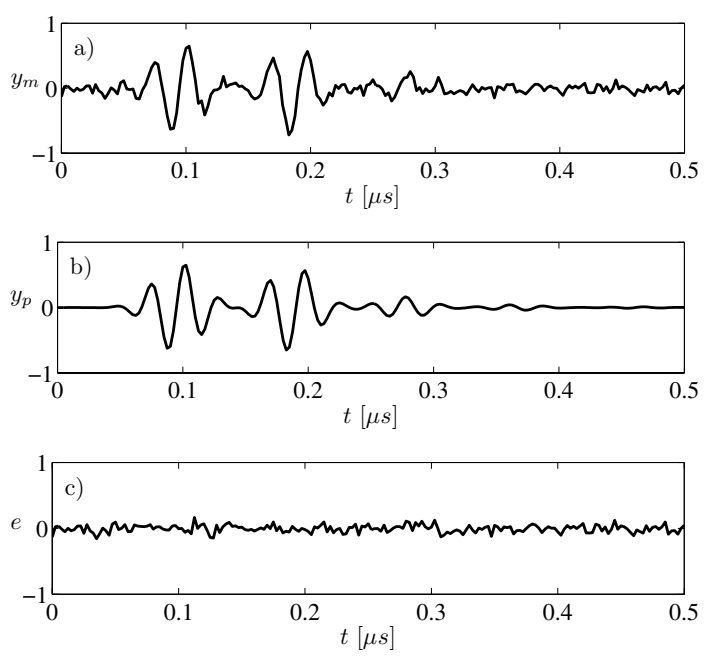

Fig. 3. a) The simulated output signal with added measurement noise $y_{m}$, $\mathrm{SNR}=10$. b) The estimated output signal $\left.y_{p} . \mathrm{c}\right)$ The error of the estimation, $e=y_{m}-y_{p}$.

A way of summarizing the performance of the detector is to plot the probability of detection, $P_{D}$, versus the probability of false alarm, $P_{F A}$. This type of evaluation is called the Receiver Operating Characteristics (ROC) and for this detector it is shown in Fig. 4 for different SNRs. Choosing the desired level of significance of the test, $\alpha$, will give the value of the threshold $\gamma$ that will be used in the detection algorithm. Calculating the integral in Eq. (23) with a significance level (probability of false alarm) of $\alpha=5 \%$ for varying SNR gives the threshold $\gamma$. Table I shows the values of the estimated $P_{D}$ and the threshold $\gamma$ for varying SNR for $\alpha=P_{F A}=5 \%$.

TABLE I

IN THIS TABLE THE ESTIMATED $P_{D}$ AND THE THRESHOLD $\gamma$ FOR VARYING SNR FOR $P_{F A}=5 \%$ IS PRESENTED

\begin{tabular}{|c|c|c|}
\hline SNR $[\mathrm{dB}]$ & $P_{D}[\%]$ & $\gamma$ \\
\hline 20 & 100 & $2 \cdot 10^{-29}$ \\
15 & 96.9 & $4 \cdot 10^{-8}$ \\
10 & 46.1 & 0.063 \\
5 & 13.0 & 0.422 \\
0 & 7.0 & 3.32 \\
\hline
\end{tabular}

In Table I it is shown that for SNR around $15 \mathrm{~dB}$ the probability of detection, $P_{D}$, is very high, when the $P_{F A}$ is only $5 \%$. In many NDE applications SNR around $15 \mathrm{~dB}$ is not unusual, which implies that a $\gamma$ close to zero can be considered in this case.

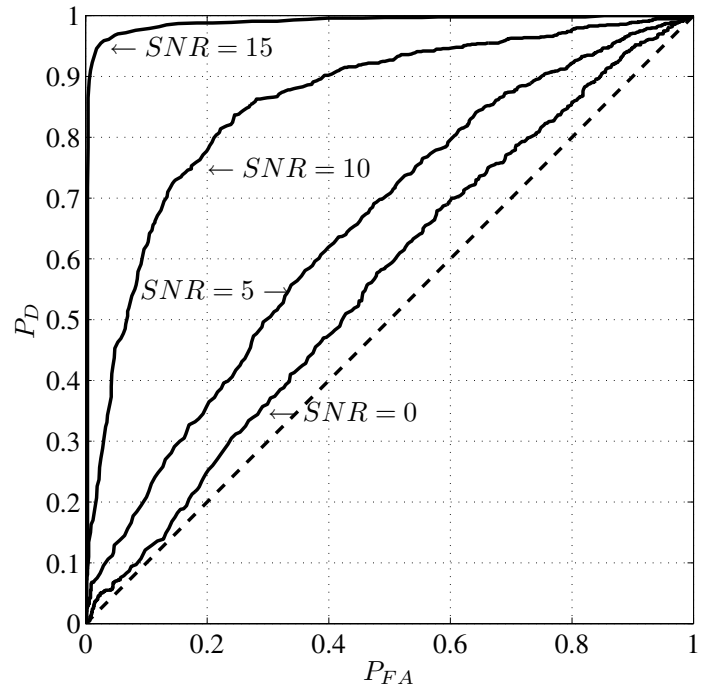

Fig. 4. Receiver operating characteristics for four different SNR, $0 \mathrm{~dB}, 5$ $\mathrm{dB}, 10 \mathrm{~dB}$ and $15 \mathrm{~dB}$. The dashed line show when there is equal probability for a correct detection and to get a false alarm.

\section{CONCLUSIONS}

The parametric model presented in this paper is able reconstruct the ultrasonic signal waveform consisting of multiple overlapping echoes from within multi-layered structures, by using the estimated parameter vector. Given the model it is possible to extract material properties of the individual layers.

We also demonstrated that a detector based on the GLRT performed on the model parameters is able to detect flaws in such structures.

\section{REFERENCES}

[1] E. P. Papadakis, "Online Statistical Process Control with NDE and Computers," in Proc. IEEE Int. Ultrason. Symp., (Chicago, IL, USA), pp. 523-527, IEEE, Oct. 2-5 1988.

[2] J. S. Heyman, W. P. Winfree, F. R. Parker, D. M. Heath, and C. S. Welch, "Quantitative NDE Applied to Composites and Metals," in Nondestructive Monitoring of Materials Properties Symposium, (Boston, MA, USA), pp. 211-220, Mater. Res. Soc, Nov. 23-30 1983.

[3] R. Mehrabian and N. G. Wadley, "Needs for Process Control in Advanced Processing of Materials," J. Met., vol. 37, no. 2, pp. 51-58, 1985.

[4] J. Makhoul, "Linear Prediction: A Tutorial Review," Proc. IEEE, vol. 63, no. 4, pp. 561-580, 1975.

[5] T. Wang, J. Saniie, and X. Jin, "Analysis of Low-Order Autoregressive Models for Ultrasonic Grain Signal Characterization," IEEE Trans. Ultrason. Ferroelectr. Freq. Control, vol. 38, no. 2, pp. 116-124, 1991.

[6] L. E. Kinsler, A. R. Frey, A. B. Coppens, and J. V. Sanders, Fundamentals of Acoustics. New York: Wiley, 3rd ed., 1982.

[7] L. M. Brekhovskikh, Waves in Layered Media, vol. 2. Academic Press, 1980.

[8] R. Pintelon and J. Schoukens, System Identification: A Frequency Domain Approach, vol. 1. IEEE Press, 2001.

[9] S. M. Kay, Fundamentals of Statistical Signal Processing: Estimation Theory, vol. 1. Prentice Hall, 1993.

[10] S. M. Kay, Fundamentals of Statistical Signal Processing: Detection Theory, vol. 1. Prentice Hall, 1998.

[11] R. Demirli and J. Saniie, "Model-Based Estimation of Ultrasonic Echoes. Part I: Analysis and Algorithms," IEEE Trans. Ultrason. Ferroelectr. Freq. Control, vol. 48, no. 3, pp. 787-802, 2001. 